\title{
Rudiments of IsoGravitation for Matter and its IsoDual for AntiMatter
}

\author{
Ruggero Maria Santilli \\ Thunder Fusion Corporation, Tarpon Springs, U.S.A
}

Email address:

research@thunder-energies.com

\section{To cite this article:}

Ruggero Maria Santilli. Rudiments of IsoGravitation for Matter and its IsoDual for AntiMatter. American Journal of Modern Physics. Special Issue: Issue I: Foundations of Hadronic Mathematics. Vol. 4, No. 5-1, 2015, pp. 59-75. doi: 10.11648/j.ajmp.s.2015040501.18

\begin{abstract}
In this paper, we hope to initiate due scientific process on some of the historical criticisms of Einstein gravitation expressed by Einstein himself as well as by others. These criticisms have remained widely ignored for one century and deal with issues such as: the apparent lack of actual, physical curvature of space due to the refraction of star-light within the Sun chromosphere; the absence of a source in the field equations due to the electromagnetic origin (rather than the charge) of gravitational masses; the lack of clear compatibility of general relativity with special relativity, interior gravitational problems, electrodynamics, quantum mechanics and grand unifications; the lack of preservation over time of numerical predictions inherent in the notion of covariance; and other basic issues. We show that a resolution of these historical doubts can be apparently achieved via the use of the novel isomathematics and related iso-Minkowskian geometry based on the embedding of gravitation in generalized isounits, with isodual images for antimatter. Thanks to half a century of prior research, we then show that the resulting new theory of gravitation, known as isogravitation, preserves indeed Einstein's historical field equations although formulated on the iso-Minkowskian geometry over isofields whose primary feature is to have null isocurvature. We then show that isogravitation allows: Einstein field equations to achieve a unified treatment of generally inhomogeneous and anisotropic, exterior and interior gravitational problems; the achievement of a clear compatibility with 20th century sciences; the achievement of time invariant numerical predictions thanks to the strict invariance (rather than covariance) of gravitation under the Lorentz-Santilli isosymmetry; the apparent achievement of a consistent representation of the gravitational field of antimatter thanks ti the isodual iso-Minkowskian geometry; the apparent achievement of a grand unification inclusive of electroweak and gravitational interactions for matter and antimatter without known causality or structural inconsistencies; and other advances. We then present, apparently for the first time, the isogravitational isoaxioms characterized by the infinite family of isotopies of special relativity axioms as uniquely characterized by the Lorentz-Santilli isosymmetry which are applicable to both exterior and interior isogravitational problems of matter with their isodual for antimatter. We finally show, also for the first time, the apparent compatibility of isogravitation with current knowledge on the equivalence principle, matter black holes and other gravitational data.
\end{abstract}

Keywords: Gravitation, Isogravitation, Antimatter

\section{Introduction}

The author has stated several times in his writings that the theory developed by Lorentz [1], Poincaré [2], Einstein [3], Minkowski [4] and others, known as special relativity, has a majestic axiomatic structure and an impeccable body of experimental verifications under the conditions clearly stated by Einstein, namely, for: A) point-particles and electromagnetic waves; B) propagating in vacuum; and C) when referred to an inertial reference frame.

Whenever any of Einstein's conditions A), B), C) are violated, special relativity is at best approximately valid, and often it is completely inapplicable (rather than violated), in the sense that it produces no quantitative description at all, as it is the case for the synthesis of the neutron from the hydrogen in the core of a star for which any use of Dirac's equation has no scientific meaning [5].

By contrast, the author has stated various times that Einstein general relativity [6] is a scientific religion at this writing because of historical insufficiencies, some of which identified by Einstein himself, such as lack of clear compatibility of general relativity with special relativity, interior gravitational problems, electrodynamics, quantum mechanics and grand 
unifications, which insufficiencies have remained unaddressed by the "mainstream physics" for one full century, let alone resolved in peer reviewed journals [7] (see also the view by the late J. V. Kadeisvili [8] and papers quoted therein).

In this paper, the author reports half a century of research toward a resolution of the historical insufficiencies of general relativity via the use of a basically new mathematics and its ensuing new physical vistas in the origin of gravitation, besides its description, for the exterior and interior gravitational problems of matter and antimatter.

It should be noted that the literature accumulated in the field is very large. To avoid a prohibitive length, we only list the references of direct relevance to the problems addressed. A comprehensive presentation and list of references up to 2011 is available in the independent general review [41] with the suggestive title of New Sciences for a New Era.

\section{Historical Insufficiency of General Relativity}

\subsection{First Historical Insufficiency of General Relativity: Ignoring the Refraction of Star-light Passing Through the Sun Chromosphere, with Consequential Lack of Evidence that Space is Actually, Physicallys Curved}

As it is well known, the conjecture of an actual, physical, curvature of space was inferred from the 1.75 arc-second "bending" of star-light passing near the Sun. Half of this value, 0.87 arc-seconds, is known to be due to a purely Newtonian attraction of light.

To see it, we first recall that for Newton gravitation to be "universal" it must also attract light, and that the source of gravitation is the energy of a body since mass is a measure of our ignorance on inertia. Hence, the author always wrote Newton's equation in the identical form in terms of the energy rather than mass

$$
F=g \frac{m_{1} m_{2}}{r^{2}}=G \frac{E_{1} E_{2}}{r^{2}}, G=\frac{g}{c^{4}} .
$$

The calculation of the 0.87 arc-seconds deviation caused by Newton gravitation of star light passing near the Sun surface is then a good exercise for graduate students in physics by computing the energy equivalence $E_{1}=m c^{2}$ of the Sun, and using the energy $E_{2}=h v$ for a given frequency of visible light.

The remaining 0.87 arc-seconds deviation have been known for a century, not to be due to the curvature of space, but to the refraction of sta-light when passing through the Sun chromosphere (see, e.g., Ref. [10] and references quoted therein). Additionally, the refraction of light passing through gaseous media is inherent in the experimental confirmations of Santilli IsoRedShift (IsoBlueShift) of light traveling through cold (hot) gases [11-15] (see Figures 1, 2, 3).

Irrespective of the above, the conjecture of curvature of space has been unable to represent without ambiguities truly basic gravitational events, such as the free fall of masses that has to be necessarily along a "straight" radial line, the weight of bodies in a gravitational field, and other basic events that are clearly represented by Newton gravitation.

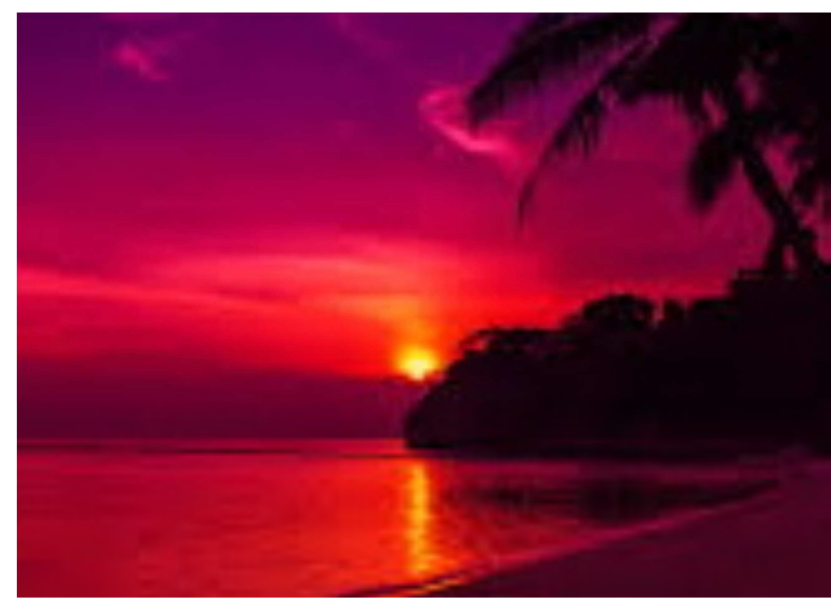

Figure 1. According to the first and perhaps most important unresolved historical criticism of Einstein gravitation, Sunset is a visual evidence of the lack of actual, physical, curvature of space because we still see the Sun at the horizon, while in reality it is already below the horizon due to the refraction of light passing through our atmosphere. Exactly the same refraction without curvature of space occurs for star-light passing through the Sun chromosphere, in which case the only "bending of light" is that due to Newton's gravitation in a flat space (see Section 2). Note that Einstein gravitation cannot represent light refraction because it requires a locally varying speed of light within a medium, first with increasing and then decreasing density. Hence, the representation of refraction via the curvature of space violates visual evidence, physical laws and experimental data [111-15]. To achieve a credible proof that the bending of Star-light passing near the Sun is "evidence" of the curvature of space, Einstein supporters have to prove that star-light passing through the Sun chromosphere does not experience refraction. The impossible existence of such a proof is readily seen from the fact that Einstein gravitation was solely aimed at a description of "exterior gravitational problems in vacuum," while the propagation of star-light within the Sun chromosphere is strictly an "interior gravitational problem" treated later on in Section 5. Its description via the Riemannian geometry is beyond any realistic possibilities due to the need for a metric possessing a dependence on coordinates $x$, as well as density $\mu$, temperature $\tau$, frequency $\omega$, etc. $g=g(x, \mu, \tau, \omega, \ldots)$ (see Sections $5-11$ below).

Despite one century of studies, the "actual" orbits of planets in our Solar system have not been represented in an accurate, unique and time invariant way via Einstein gravitation, while they are exactly and unambiguously represented by Newton's gravitation and Kepler's laws. In fact, calculations based on the Riemannian geometry of the actual orbits of planets, besides not being unique due to the non-linearity of the theory, are generally different than physical orbits, and are not the same over time (see below).

It should also be indicated that a concrete visualization of the curvature space require an increase of the number of space dimension. In fact, the curvature in a two-dimensional Riemannian space can only be seen in three dimensions, as well known. Consequently, a concrete visualization of the curvature of space in three dimensions requires the implausible assumption of a fourth space dimension.

Needless to say, gravitational waves [6] crucially depend on the curvature of space represented via the Riemannian geometry. Until we dismiss in peer reviewed journals the mathematical, theoretical, experimental and visual evidence against the curvature of space, studies on gravitational waves 
may well remain in suspended animation.

It goes without saying that a critical inspection of the conjecture of curvature of space creates great emotions in colleagues who have spent their research life on curved spaces. Yet, serious appraisals should be voiced only after identifying the huge limitations caused by curvature and only after inspecting the vast advances permitted by novel theories of gravitation on a flat space treated with the appropriate novel mathematics (Section 5).

\subsection{Second Historical Insufficiency of General Relativity: Ignoring the Electromagnetic Origin of the Mass, with Consequential Invalidation of Einstein's Reduction of Gravitation to Pure Curvature Without Sources}

As it is well known, the contribution to gravity of the total electric and magnetic field of a body is of the order of $10^{-30}$ or smaller. Consequently, following the assumption of the curvature of space, Einstein was forced to avoid any source in the r.h.s. of his field equations and reduce gravitation to pure geometry according to the the celebrated equations

$$
G_{i j}=R_{i j}-g_{i j} R / 2=0, i, j=1,2,3,4
$$

In 1974, the author identified the electromagnetic origin of the mass via the full use of quantum electrodynamics, including advanced and retarded treatments, and showed that such an origin requires the necessary presence in the r.h.s. of the field equations of a source first order in magnitude, irrespective of whether the body is charged or neutral [16],

$$
G_{i j}=R_{i j}-g_{i j} R / 2=\mathrm{kT}_{i j, e l m},
$$

where $\mathrm{k}$ is a unit-dependent constant, and the terms "first order in magnitude" are referred to the condition of entirely representing the gravitational mass of the body considered [16]

$$
\mathrm{m}_{\text {grav }}=\int \mathrm{T}_{00} \mathrm{dv} .
$$

The most skeptical physicist should admit that the mass of the electron is of entire electromagnetic origin. Therefore, field equations (2) are insufficient to represent the gravitational field of the electron in favor of Eqs. (3)-(4).

But then, the same skeptical physicist should admit that exactly the same conclusion holds for the positronium, namely, the gravitational mass of the positronium is of entire electromagnetic origin despite the total charge and magnetic moment being null. Therefore, Einstein's field equations (2) are insufficient for the representation of the gravitational field of the positronium in favor of broader Eqs. (3)-(4).

Paper [16] essentially extended the above known reality to the $\pi^{0}$-meson under the assumption of being a bound state of a charged constituent and its anti-particle. Paper [16] then extended the results to all masses with null total charge and null total magnetic moments. The inclusion of gravitational contributions from total electromagnetic characteristics was trivial.
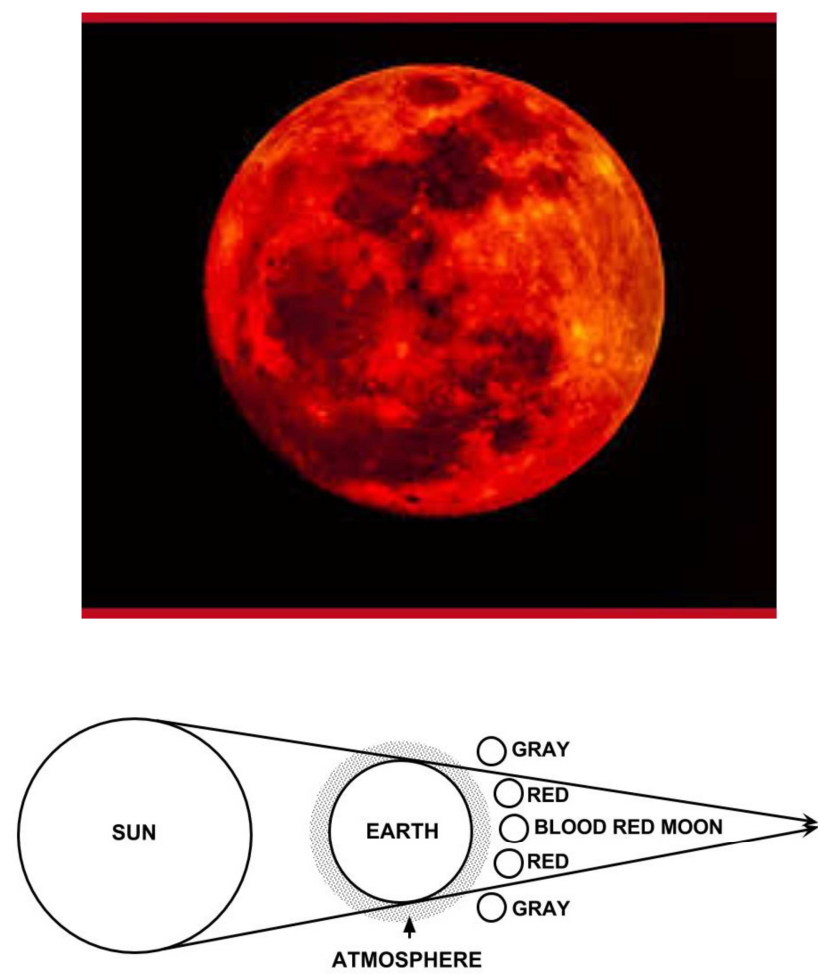

Figure 2. The "blood red moon" (top view) during a Lunar eclipse is an additional visual evidence of the lack of curvature of space because Sunlight reaches the Moon even when it should be in total darkness (bottom view). Note that for both Sunsets and Lunar eclipses the entire spectrum of Sunlight is redshifted without relative motion, merely due to loss of energy by light to a cold medium (IsoRedShift). Note also that we are dealing with "direct Sunlight" traveling in empty space for which scattering and other interpretations have been dismissed in peer refereed journals [11-15]. Note finally that the "blood red moon" confirms the view by Einstein, Hubble, Fermi, Zwicky, Hoyle, de Broglie and others on the lack of expansion of the universe because, when our Sun is seen millions of light years away, we merely have the replacement of Earth's atmosphere with very cold intergalactic gases under which the entire spectrum of visible Sunlight will appear redshifted without any relative motion [11-15].

In defense of Einstein, we have to recall that, contrary to his followers, Einstein always expressed serious doubts of field equations (2), for instance, by calling their r.h.s. A house made of wood, compared to the 1.h.s. which he called $A$ house made of marble. It is unfortunate for scientific knowledge that Einstein's own doubts have remained vastly ignored in the "mainstream literature" in gravitation.

We should also recall that, according to Ref. [16], the characterization of the inertial mass of a body requires the additional inclusion of all possible short range (e.g., weak and string) interactions, resulting in the need for an additional source in the r.h.s. of the equations whenever considering interior gravitational problems

$$
G_{i j}=R_{i j}-g_{i j} R / 2=k_{1} T_{i j, e l m}+k_{2} T_{i j, s h o r t r a n g e},
$$

such that $(\mathrm{c}=1)$

$$
\mathrm{m}_{\text {inert }}=\int\left(\mathrm{T}_{00, \mathrm{elm}}+\mathrm{T}_{00, \text { shortrange }}\right) \mathrm{dv}
$$

Consequently, the inertial mass is predicted as bigger than 
the gravitational mass $[16](\mathrm{c}=1)$

$$
\mathrm{m}_{\text {inert }}>\mathrm{m}_{\text {grav }}
$$

The expectation is that serious scientists will admit our lack of final experimental resolution on the relationship between the exterior gravitational and the interior inertial mass.

Besides the incontrovertible need for a source of first order in magnitude, the structure of Eqs. (5)-(6) is mandated by the fifth identity of the Riemannian geometry, the forgotten Freud identity [17] (see also the recent treatment by the late mathematician H. Rund [18]) which establishes the need on purely mathematical grounds of a source of first order in magnitude in the r.h.s of the field equations according precisely to Eqs. (5)-(6).

In fact, the source term of the Freud identity can be decomposed into a term with null trace, (evidently, the electromagnetic term), and a term with non-null trace (evidently, the source for short range interactions), thus providing a geometric confirmation of Eqs. (5)-(6).

We should indicate that the problem of a source in the gravitational field equations has been debated at length in the literature (see, e.g., Ref. [6]), although for its interpretation as a stress-energy tensor, or for other interpretations, while generally ignoring its electromagnetic origin.

Interested scholars should be aware of various claims in the literature that Einstein's gravitation verifies the Freud identity. These claims are based on the admission indeed of a source of electromagnetic nature, but restricted to the the total electromagnetic characteristics, thus violating condition (4) by a missing factor of $10^{30}$ or so.

Additionally, and perhaps more importantly, the Freud identity requires a source of first order in magnitude also for bodies with null total electromagnetic characteristics, thus confirming the lack of compliance of Einstein gravitation with the Freud identity.

Remember that gravitational waves are crucially dependent on Einstein's reduction of gravitation to pure geometry, Eqs, (2) [6]. However, physical and geometric needs mandate their extension to Eqs. (3), (4), for which gravitational waves cannot even be formulated, to our best knowledge at this writing.

Therefore, by noting the lack of independent addressing of the issues for the last four decades since the appearnce of paper [16], the theoretical prediction of gravitational waves will remain in suspended animation until the additional problem of theelectromagnetic origin of the gravitational mass is dismissed in refereed publications.

Again, the author has experienced over decades huge emotional reactions by colleagues at the instant of examining Einstein's reduction of gravitation to pure geometry, Eq. (2), without any in depth inspection of the advances permitted by a source term as in Eqs. (3)-(4). In a nutshell, the alternative between Eqs. (2) and (3), (4) bolls down to the belief of the existence of local infinities in the universe or not. Eqs. (2) do admit these local infinities, while covering Eqs. (3), (4) recover all main results of Eqs. (2) except replacing local infinities with large, yet finite values (Section 5 and Subsection 5.10 in particular).

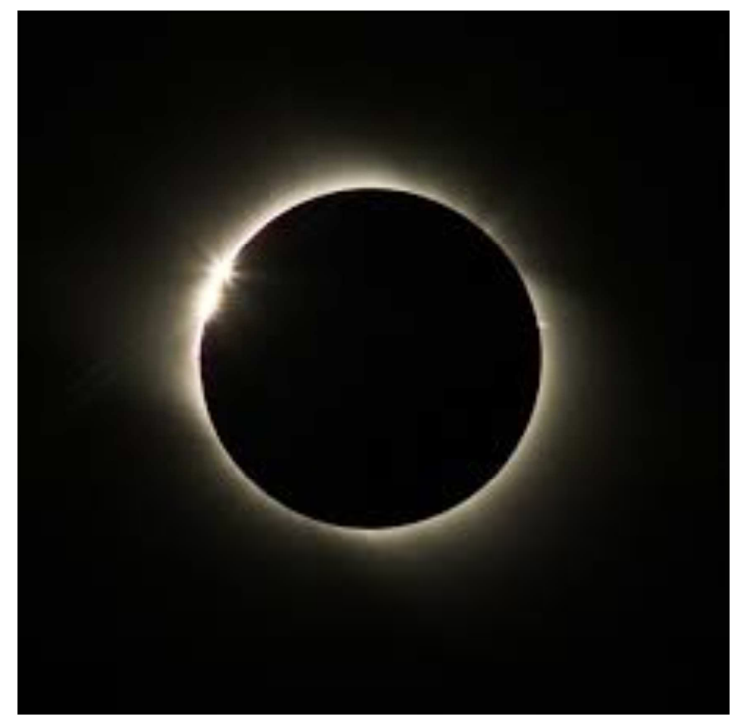

Figure 3. A view of a Solar eclipse showing no "bending of light" because the Newtonian attraction of light by the moon is extremely small and there is no refraction due to the lack of lunar atmosphere. The faint luminescence at sea level is due to the diffraction of light in our atmosphere. In conclusion, final claims of "bending of light due to curvature of space" must be based on star light passing tangentially on a body without atmosphere or chromosphere and be proved to be greater than the Newtonian attraction.

As a final note, the reader may have noted the lack of use of the mathematical terms "tensors" or "oseudotensors" and the use instead of the physical term "source." This is due to the fact that the clear physical content of the forgotten Freud identity is often dismissed on ground of purely mathematical differences in nomenclatures and personal mathematical interpretations without serious physical implications.

\subsection{Third Historical Insufficiency of General Relativity: Abandoning the Majestic Lorentz and Poincaré "Invariance" of Special Relativity in Favor of the "Covariance" of General Relativity with Consequential Lack of Prediction of the Same Numerical Values under the Same Conditions at Different times}

In our view, the above is perhaps the biggest insufficiency of Einstein gravitation because it implies the inability of gravitation to have time invariance, here referred to the prediction of the same numerical values under the same conditions at different times, while such a crucial requirement is verified by Galileo relativity and Einstein special relativity because of their Galilei and Poincare' symmetries, respectively.

In turn, the lack of time invariance establishes the lack of final character of all claims of "experimental verification of general relativity" [9] due to the absence of a physically consistent dynamical evolution.

In fact, "experimental verifications" of general relativity are done in ad hoc selected coordinate systems generally with no connection to the frame of the experimenter, thus prohibiting final experimental values, not only because said systems are different among themselves, but also because the needed experimental frame is generally not necessarily achievable via covariance. 


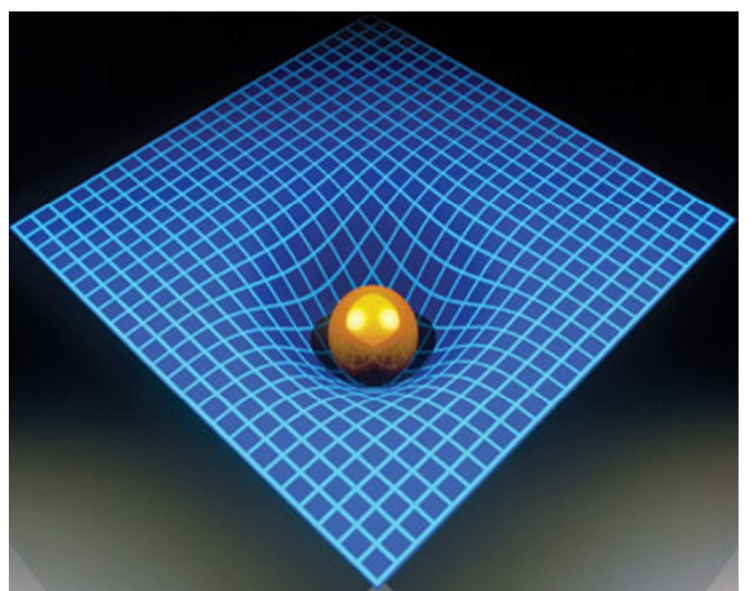

Figure 4. A typical representation of the claimed curvature of space caused by the gravitational field of a mass, which representation has been adopted for one full century. The historical, yet unresolved criticism is that the notion of physical curvature in one dimension requires a bigger dimension for its identification. In fact, the physical interpretation of the mathematical Riemannian curvature in two dimension can only be identified in three dimension as clearly illustrated by the above figure. Therefore, the additional historical criticism of Einstein gravitation that needs to be addressed is that the physical identification of the mathematical Riemannian curvature in three dimensions, as needed for realistic models of gravitations, requires four space dimensions that do not exist, thus confirming the lack of physical evidence for the actual physical curvature of space depicted in Figure 1, 2, 3. In any case, Einstein supporters are requested to illustrate with concrete geometric example the physical curvature needed for realistic models, not in two dimensions as done for one century, but in three dimensions.

Under the lack of invariance, general relativity could at best offer a kind of "polaroid picture" of gravitation [7,8]. However, such a static view of gravitation is dismissed by mathematical, physical, visual and experimental evidence on the lack of existence of the actual curvature of space.

Additional rather serious objections against published claims of "experimental verifications of Einstein gravitation" [9] stem from the fact that numerical predictions are, by far, not unique and/or unambiguous due to the non-linearity of the field equations. In fact, for any claim of "experimental verification" [9] we can assume a different PPN approximation with different expansions and show dramatic divergences with physical realities $[7,8]$.

The lack of time invariance of Einstein's gravitation identifies an additional impossibility for gravitational waves to exist because any serious experimental verification should not only detect gravitational waves, which has been impossible for half a century despite the use of large public funds, but said gravitational waves should change in time without any change of the source, which is a blatant physical impossibility.

In defense of Einstein we should indicate that, once the Riemannian geometry is assumed for the representation of gravitation, no symmetry of the line element is possible for technical reasons similar to those of the historical Lorentz problem. We are here referring to Lorentz inability to achieve the invariance of the locally varying speeds of light of his time, that within physical media $\mathrm{C}=\mathrm{c} / \mathrm{n}$, due to insurmountable technical difficulties in attempting to use Lie's theory for non-linear systems.

This is yet another case in which the author has experienced pre-judgments by colleagues mainly due to decades of research with covariance in gravitation without a serious inspection of qualified alternative views. In reality, serious judgments can only be expressed after a technical knowledge of the huge possibilities for further advances in gravitation permitted by alternative invariant theories (Section 5).

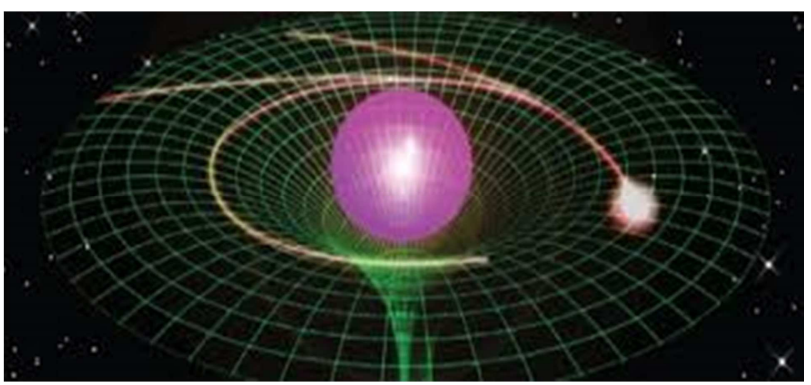

Figure 5. I Another illustration of the insufficiencies of the one century old assumption that planets moving around the Sun in our Solar system actually move along a real, physical curvature of space. The historical criticism is that the above representation is purely mathematical because, to actually sense curvature in a three-dimensional space, the planet should move in a fort space dimension that does not exist.

\subsection{Consequences of the Historical Insufficiencies of General Relativity: Incompatibility of Gravitation with Special Relativity, Interior Gravitational Problems, Electrodynamics, Quantum Mechanics, and Grand Unifications}

There comes a point in the life of a scientist in which realities have to be admitted. The Riemannian geometry does indeed admit a unique and unambiguous reduction to the Minkowskian geometry via tangent, limit and other procedures.

However, it has been known for a century that general relativity does not admit a clear and unambiguous limit to special relativity of the type according to which special relativity uniquely and unambiguously admits a limit into the Galilei relativity. As one among many impossibilities, there exists no consistent limit of the covariance of general relativity into the fundamental Poincaré invariance of special relativity. The inconpatibilities that follow are then endless.

Another serious insufficiency is that the description by general relativity of "exterior gravitational problems" in vacuum is incompatible with "interior gravitational problems" that dominated the scientific scene in gravitation until the advent of Einstein's theory (e.g., Schwartzchild wrote two papers, one on the exterior and one on the interior gravitational problem [6], the second one being vastly ignored).

This is a serious incompatibility because its resolution prohibits the use of the Riemannian geometry due to the need of a geometry not only without curvature, but also (as indicated in Fig.1) with a metric having a dependence on coordinates $\mathrm{x}$, as well as density $\mu$, temperature $\tau$, frequency $\omega$, etc. $g=g(x, \mu, \tau, \omega, \ldots)$ (see Section 5 for details).

Another aspect that should be admitted to prevent exiting from physical reality is the irreconcilable incompatibility between Einstein gravitation and electrodynamics to such an extent that [16]: 
2A) Either one assumes Einstein's gravitation as being valid, in which case electrodynamics must be revised from its foundations so as to eliminate the electromagnetic origin of the mass, or

2B) One assumes electrodynamics and its inherent origination of the gravitational mass as being valid, in which case, Einstein gravitation must be revised from its foundations.

Yet another reality that has to be faced following one century of wide oblivion, is that Einstein's gravitation is incompatible with quantum mechanics as conventionally understood, (that is, a unitary theory on a Hilbert soace) for several reasons. The reason most important in our view is that a gravitational theory formulated on a Riemannian space is necessarily non-canonical at the classical level (variationally non-self-adjoint [20]).

Therefore, any consistent "quantization" of Einstein gravitation must be non-unitary, with the consequential activation of the Theorems of Catastrophic Inconsistencies of Non-Canonical and Non-Unitary Theories [19] and ensuing loss of physical value, e.g., due to the violation of causality laws.

The moment of truth also implies the admission that Einstein gravitation is incompatible with grand unified theories, if nothing else, because of failed attempts o [6]ver one full century, beginning with the failed attempt of unifying gravitation and electromagnetism by Einstein himself.

\subsection{Problems to be Solved for an Axiomatically Consistent Grand Unification}

Following studies on grand unifications for decades, the incompatibilities of a grand unification of Einstein gravitation with electroweak interactions are the following (see, later on monograph [40]):

2.I. The physical consistency of electroweak interactions on a flat Minkowski space cannot be salvaged when joined to a theory on the curved Riemannian space because the insufficiencies of the latter carry over to the former;

2.II. Within a grand unification, the covariance of Einstein's gravitation carries over to electroweak interactions, by therefore destroying their gauge invariance and,consequently, the very structure of electroweak interactions;

2.III. Electroweak interactions represent both particles and antiparticles, while Einstein gravitation solely represent matter, thus rendering any grand unification technically impossible and catastrophically inconsistent if attempted.

We should mention a recent trend of extending the applicability of special and general relativities to the classical representation of antimatter. Serious scholars should be alerted that this trend is afflicted by serious inconsistencies, such as the impossibility of admitting the annihilation of matter and antimatter precisely due to the lack of a conjugation in the transition from matter to antimatter, violation of the PCT theorem and other inconsistencies.

Another reality that should be faced by serious scholars in the field is that a consistent representation of the gravitational field of antimatter cannot be achieved by Einstein gravitation and a new theory must be constructed from its mathematical foundations.

\section{Rudiments of IsoMathematics}

The most important lesson the author has learned in fifty years of research is that the protracted lack of resolution of physical problems is generally due to the use of insufficient mathematics, rather than to physical issues.

We believe that this is precisely the case for gravitation, namely, all problems treated above are caused by the use of an excessively insufficient mathematics, that based on the differential calculus that dates back to the Newton-Leibniz times. Only after the achievement of a more adequate mathematics, open physical problems can be quantitatively and effectively addressed.

To see the case, note that for a theory of gravitation to resist the test of time, it is expected to possess an invariance similar to that of the Poincaré symmetry in special relativity so as to predict the same numerical values under the same conditions at different times.

The best known way to achieve an invariant theory of gravitation is via the use of Lie's theory. But the latter theory solely applies to linear systems. The necessary non-linearity of gravitation then precludes any realistic possibility of achieving an invariance via the use of 20th century mathematics.

The above occurrence forced the author to construct the isotopies (intended as axiom-preserving) of 20th century applied mathematics [20], today known as isomathematics, that was initiated by when author was at the Department of Mathematics of Harvard University in the late 1970s under DOE support.

Isomathematics is based on the isotopic lifting of the conventional associative product $\mathrm{AB}$ between generic quantities A, B (such as numbers, functions, matrices, etc.) into the isoproduct [19b]

$$
A \widehat{x} B=A \widehat{T} B
$$

where the quantity $\widehat{T}$, called the isotopic element, is positive definite but otherwise posses an arbitrary functional dependence on all needed local quantities, such as time $t$, coordinates $\mathrm{r}$, velocities $\mathrm{v}$, accelerations $\mathrm{a}$, density $\mu$, temperature $\tau$, frequency $\omega$, wavefunction $\psi$, etc. $\widehat{\mathrm{T}}=$ $\widehat{\mathrm{T}}(\mathrm{t}, \mathrm{r}, \mathrm{v}, \mathrm{a}, \mu, \tau, \omega, \psi, \ldots)>0$.

Product (8) was introduced for the primary intent of achieving an invariant representation of interior dynamical problems referred to extended, non-spherical and deformable particles moving within physical media, which is notoriously impossible via 20th century mathematics, but possible via isomathematics (see below for examples).

Therefore, isomathematics was suggested for the primary intent of achieving a generalization of Lie's theory into a form applicable for the first time to non-linear, non-local and non-Hamiltonian systems (that is, variationally nonself-adjoint systems not representable with a Hamiltonian [20a]).

A systematic isotopic lifting of the various branches of Lie's theory was presented in monograph [20b]. The resulting theory is today known as the Lie-Santilli IsoTheory, and it is 
based on the isoalgebra (the isotopues of Lie's second theorem)

$$
\left[\mathrm{X}_{\mathrm{i}}, \mathrm{X}_{\mathrm{j}}\right]=\mathrm{X}_{\mathrm{i}} \widehat{\times} \mathrm{X}_{\mathrm{j}}-\mathrm{X}_{\mathrm{j}} \widehat{\mathrm{X}} \mathrm{X}_{\mathrm{i}}=\mathrm{X}_{\mathrm{i}} \widehat{\mathrm{T}} \mathrm{X}_{\mathrm{j}}-\mathrm{X}_{\mathrm{j}} \widehat{\mathrm{T}} \mathrm{X}_{\mathrm{i}}=\mathrm{iC}_{\mathrm{ij}}^{\mathrm{k}} \mathrm{X}_{\mathrm{k}}
$$

and their integration into a finite isogroup here illustrated for simplicity via the one dimensional time evolution with the Hamiltonian $\mathrm{X}=\mathrm{H}$

$$
A(t)=e^{X \widehat{T} t i} A(0) e^{-i t \widehat{T} X}
$$

with evident non-linear, non-local and non-Hamiltonian characters due to the presence of the isotopic element in the exponent.

In Vol. [20b], the author then presented a concrete realization of the Lie-Santilli isotheory given by the Birkhoffian generalization of classical Hamiltonian mechanics and its "direct universality," namely, the representation of all infinitely possible, well behaved, non-Hamiltonian systems directly in the frame of the experimenter.

However, the new mechanics activated the Theorems of Catastrophic Inconsistencies of Non-Canonical and Non-Unitary Theories when formulated via the mathematics of canonical and unitary theories, respectively [19].

Therefore, while visiting at the JINR in Dubna, Russia, the author was forced in 1993 [21] to reinspect the historical classification of numbers and discovered that the abstract axioms of a numeric field do not necessarily require that the basic multiplicative unit is the number +1 , since they also admit realizations with arbitrary positive-definite units, provided that the associative product is lifted accordingly.

This lead to the discovery of new numbers, today known as Santilli isonumbers, with an arbitrary positive-=definite unit, called Santilli isounit, which is the inverse of the isotopic element of isoproduct (8)

$\hat{\mathrm{I}}(t, r, v, a, \mu, \tau, v, \psi, \ldots)=1 / \widehat{T}(t, r, v, a, \mu, \tau, v, \psi, \ldots$.

Applied mathematics was then reformulated on isofields. Yet, the fundamental invariance under the time evolution remained elusive. This forced the author to lift the Newton-Leibniz differential calculus into the form today known as Santilli IsoDifferential Calculus first presented in mathematical memoir [22] of 1996, with basic isodifferential

$$
\hat{d} \hat{r}=d r+r \hat{T} d \hat{I}
$$

and related isoderivative

$$
\frac{\hat{\partial} \hat{F}(\hat{r})}{\hat{\partial} \hat{r}}=\hat{I} \frac{\partial \hat{F}(\hat{r})}{\partial \hat{r}},
$$

where the realizations $\hat{F}=F \hat{I}, \hat{r}=r \hat{I}$, etc. are necessary for the values to be isonumbers.

The isodifferential calculus permitted the achievement of maturity for mathematical, physical, and chemical developments, with ensuing numerous scientific as well as industrial applications. Isomathematics is today referred to the isotopies of the totality of $20^{\text {th }}$ century mathematics formulated via isofunctional analysis, isodiufferential calculus, isoalgebras, isosymmetries, isogeometriesc, etrc., on Santgilli isofields.

A comprehensive presentation of isomathematics for physicists has been provided by the author in monographs [23]. A presentation of isomathematiccs for mathematicians is available in monograph [23] by R. M. Falcon Ganfornina and J. Nunez Valdes, while a monumental work on the isodifferential calculus and its bimplications for all of mathematics is available in the five monographs [25] by $\mathrm{S}$. Georgiev.

\section{Rudiments of IsoMechanics}

The primary physical application of isomathematics is the isotopic lifting of Newton's equations, first presented in Ref. [22]

$$
\widehat{m} \widehat{\times} \frac{\hat{d} \hat{v}}{\hat{d} \hat{t}}=F^{s a}(t, r, v) \cdot \text { principle }
$$

today known as the Newton-Santilli IsoEquations.

Eqs. (14) allow the first known representation of the actual extended shape of bodies, for instance, via the isounit for the velocities

$$
\begin{gathered}
\hat{I}(t, r, v, a, \mu, \tau, \omega, \ldots)=\operatorname{Diag} .\left(n_{1}^{2}, n_{2}^{2}, n_{3}^{2}\right) e^{\Gamma}, \\
n_{k}=n_{k}(t, r, v, a, \mu, \tau, v, \ldots)>0 \\
\Gamma=\Gamma(t, r, v, a, \mu, \tau, \omega, \ldots)>0, k=1,2,3
\end{gathered}
$$

as well as the representation of non-Hamiltonian (variationally non-self-adjoint [20]) forces via the exponent of the isounit (15) and their embedded in the isodifferential $\hat{d} \hat{v}=\hat{d}(v \hat{I})$ in such a way that only Hamiltonian (variationally self-adjoint [20]) forces appear in the r.h.s. of the equations.

In view of these features, the Newton-Santilli isoequations for non-Hamiltonian systems admit the first known representation via isoaction principle [22]

$$
\hat{\delta} \hat{A}=\int(\hat{p} \widehat{\times} \hat{d} \hat{r}-\widehat{H} \widehat{\times} \hat{t})=0
$$

thus permitting the first known use of the optimal control theory for the shape, e.g., of a wing moving within a fluid.

In turn, the availability of the isoaction principle has allowed the isotopic lifting of classical Hamiltonian mechanics into its covering Hamilton-Santilli isomechanics with basic isotopies of the conventional Lagrange and Hamilton equations here ignored for brevity as well as of the Hamilton-Jacobi-Santilli isoequations [22,23]

$$
\frac{\hat{\partial} \hat{A}}{\hat{\partial} \hat{t}}+\widehat{H}=0, \quad \frac{\hat{\partial} \hat{A}}{\hat{\partial} \hat{r}}-\hat{p}=0, \quad \frac{\hat{\partial} \hat{A}}{\hat{\partial} \hat{p}}=0 .
$$

Still in turn, the availability of the latter isoequations has permitted the first known, axiomatically consistent, unique and unambiguous, operator map of non-Hamiltonian systems into a covering of quantum mechanics introduced in 1978 under the name of hadronic mechanics [20], with Schrödinger-Santilli Isoequations [22] 


$$
\begin{gathered}
\hat{\imath} \widehat{\times} \frac{\hat{\partial} \hat{\psi}}{\hat{\partial} \hat{t}}=\widehat{H} \widehat{\times} \hat{\psi}= \\
=\widehat{H}(\hat{r}, \hat{p}) \widehat{T}(t, r, p, \mu, \tau, \nu, \psi, \ldots) \hat{\psi}= \\
=\hat{E} \widehat{\times} \hat{\psi}=E \hat{\psi},
\end{gathered}
$$

related isolinear momentum

$$
\hat{p} \widehat{\times} \hat{\psi}=-\hat{\imath} \widehat{\times} \frac{\partial \hat{\psi}}{\hat{\partial} \hat{r}}=-i \hat{I} \frac{\partial \hat{\psi}}{\partial \hat{r}}
$$

and their isounitarily equivalent Heisenberg-Santilli isoequations [20,23] for the isotime evolution of an operator $\hat{A}$ in the infinitesimal form

$$
\begin{aligned}
& \hat{\imath} \widehat{\times} \frac{\hat{d} \hat{A}}{\hat{d} \hat{t}}=[\hat{A} \hat{,} \widehat{H}]=\widehat{H} \widehat{\times} \widehat{H}-\widehat{H} \widehat{\times} \hat{A}= \\
& =A \widehat{T}(t, r, p, \mu, \tau, v, \psi, \ldots) \widehat{H}(\hat{r}, \hat{p})- \\
& -\widehat{H}(\hat{r}, \hat{p}) \widehat{T}(t, r, p, \omega, \tau, \omega, \psi, \ldots) A,
\end{aligned}
$$

and integrated finite form (10), where the "hat" denotes formulation on an iso-Hilbert space over the isofield of isocomplex numbers [23].

For readers not familiar with the field, we should recall that hadronic mechanics is a non-unitary "completion" of quantum mechanics much along the celebrated argument by Einstein-Podolsky and Rosen (see later on Ref. [36]). However, non-unitary theories formulated on a conventional Hilbert space over a conventional field violate causality $[9,19]$ Hence, the reformulation of non-unitary theories via isomathematics is crucial for the mathematical and physical consistency of hadronic mechanics at large and its isomechanical branch in particular (see monographs [23] for a comprehensive presentation).

We should also mention that hadronic mechanics eliminates the divergencies of quantum mechanics because the value of the isounit (15) is generally very big. Consequently, the value of the isotopic element $\widehat{T}$ is very small, thus permitting the conversion of divergent or weakly convergent quantum series into strongly convergent isotopic forms via the systematic use of isoproduct (8). Additionally, the functional dependence of the isotopic element is unrestricted, thus allowing the removal of the singularity of the Dirac delta distributions under isotopy, which feature persists for the isotopies of the scattering theory. The absence of divergencies is particularly important for approximate solutions of exterior and interior dynamical problems, as well as of non-linear gravitational equations when reformulated in terms of isomathematics.

Finally, the non-initiated reader should be aware that quantum mechanics and hadronic mechanics coincide at the abstract level by conception and construction to such an extent that they can be expressed via the same symbols and equations, merely subjected to different realizations. Following decades of research in the field, we believe that the above features are iportant to assure consistency and causality of hadronic mechanics and its applications.

\section{Rudiments of IsoGravitation for Matter}

\subsection{Elementary Formulation of IsoGravitation}

The main result of the studies in gravitation herein reported is that the conjecture of the actial curvature of space is the dominant origin of all problematic aspects of Einstein gravitation, including all its incompatibilities with 20th century sciences, besides being disproved by visual, mathematical and experimental evidence (Figure 1-5).

Therefore, the main objectives of the studies herein reported are: A) the reformulation of Einstein field equations via a basically new geometry admitting the invariance of line elements without curvature; B) show the compatibility of said reformulation with 20th century sciences; and C) provide at least preliminary experimental verifications.

Following decades of preparatory research on the new isomathematics and isomechanics, isogravitation for matter was presented for the first time at the 1992 Marcel Grossmann Meeting in Gravitation [26] via the following elementary rules:

RULE 5-I: Decompose any non-singular Riemannian metric $\mathrm{g}(\mathrm{x})$ in $(3+1)$-dimensions into the product of the the Minkowski metric $\eta=\operatorname{Diag} \cdot(1,1,1,-1)$ and the $4 \times$ 4-dimensional gravitational isotopic element $\widehat{T}_{g r}(x)$

$$
g(x)=\widehat{T}_{g r}(x) \eta
$$

where the positive-definite character of $\widehat{T}_{g r}(x)$ is assured by the topology of the Riemannian space;

RULE 5-II: Assume the inverse of the isotopic element as the gravitational isounit

$$
\hat{I}_{g r}(x)=1 / \widehat{T}_{g r}(x)>0
$$

RULE 5-III: Reformulate the totality of Einstein gravitation into such a form admitting $\hat{I}_{g r}(x)$ as the correct left and right unit at all levels, including numbers, functional analysis, differential calculus, geometries, Christoffel symbols, etc.

As we shall see, the above simple rules will allow maintaining Einstein's field equations including its primary verifications, although formulated on a new geometry over new fields with null curvature.

\subsection{Minkowski-Santilli IsoSpace}

The spacetime of isogravitation verifying the above conditions is given by the infinite family of isotopies of the Minkowski space first introduced by the author in Ref. [26] of 1983 for the classical profile and Ref. [27] of the same year for the operator counterpart, and it is today known as the Minkowski-Santilli IsoSpace.

Consider the conventioinal Minkowski space $M(x, \eta, I)$ with spacetime coordinates $x=(\mathrm{xi}), i=1,2,3,4$, metric $\eta=\operatorname{Diag} \cdot\left(1,1,1,-c^{2}\right)$ and unit $I=\operatorname{Diag} \cdot(1,1,1,1)$. The Minkowski-Santilli isospace is denoted $\widehat{M}(\hat{x}, \hat{\eta}, \hat{I})$, and it is characterized by the infinite family of isotopies for which coordinates are lifted into isocoordinates (a necessary condition for their value to be isonumbers) [26] 


$$
x \rightarrow \hat{x}=x \hat{I}
$$

the Minkowski metric is lifted into the infinite family of isometrics

$$
\eta \rightarrow \hat{\eta}=\widehat{T}_{g r} \eta
$$

the Minkowski unit is lifted into the isounits with related isotopic elements

$$
\begin{gathered}
\hat{I}_{g r}(t, r, p, \mu, \tau, \omega, \psi, \ldots)= \\
=\operatorname{Diag} \cdot\left(n_{1}^{2}, n_{2}^{2}, n_{3}^{2}, n_{4}^{2}\right)>0, n_{i}>0, \\
\widehat{T}_{g r}(t, r, p, \mu, \tau, \omega, \psi, \ldots)= \\
=\operatorname{Diag} \cdot\left(\frac{1}{n_{1}^{2}}, \frac{1}{n_{2}^{2}}, \frac{1}{n_{3}^{2}}, \frac{1}{n_{4}^{2}}\right)>0,
\end{gathered}
$$

and line Minkowski element into the infinite family of isoline elements

$$
\begin{aligned}
\hat{x}^{\widehat{2}} & =\hat{x}^{i} \widehat{\times} \hat{\Xi}_{i j} \widehat{\times} \hat{x}^{j}=\left(x^{i} \hat{\eta}_{i j} x^{j}\right) \hat{I}= \\
& =\left(\frac{x_{1}^{2}}{n_{1}^{2}}+\frac{x_{2}^{2}}{n_{2}^{2}}+\frac{x_{3}^{2}}{n_{3}^{2}}-t^{2} \frac{c^{2}}{n_{4}^{2}}\right) \hat{I},
\end{aligned}
$$

where: $\hat{\Xi}=\hat{\eta} \hat{I}$ is a condition is a condition to have correct isomatrices, that is, matrices whose elements are isonumbers; one should note the multiplication of the isoline elements by the isounit which is also a necessary condition forf the line element to be isonumbers; and we have ignored for simplicity the exponential factor in the isounits and isotopic elements representing non-Hamiltonian interactions as in Eqs. (15) (see Refs. [23] for the full treatment).

The $n$-quantities are called the characteristic quantities of the gravitational field and they are illustrated in the verifications below. Readers are suggested to exercise caution for the popular interpretation of the $n$-quantities as being "free parameters" since this would literally imply that, for instance, the terms characterizing the Schwartzchild metric are "free parameters."

It is easy to see that the projection of the isoline element (27) in conventional spacetime is the most general possible symmetric (thus diagonalized) and non-singular line element in (3+1)-dimensions, thus including as particular cases all possible Minkowskian, Riemannian, Fynslerian and other line elements (it should be noted that non-symmetric line elements for the geometric representation of irreversible gravitational events require the broader Lie-admissible genomathematics $[19,23])$

\subsection{Minkowski-Santilli IsoGeometry}

The geometry of isospace $\widehat{M}(\hat{c}, \hat{\eta}, \hat{I})$ was first studied in memoir [28] of 1998 and it is today known as the Minkowski-Santilli isogeometry. Its first important feature is the admission of the entire machinery of the Riemannian geometry, such as covariant derivative, Christoffel symbols, etc. merely reformulated in terms of the isodifferential calculus, Eqs. (12)-(13).

This is evidently due to the fact that, unlike the Minkowski metric $\eta$, its isotopic covering $\hat{\eta}$ admits the most general possible functional dependence, under the sole condition of positive-definiteness of the isotopic element, Eq. (26). Regrettably, an outline of the new geometry would be excessively advanced for the elementary character of this presentation.

The second important feature of the Minkowski-Santilli isogeometry is that of being isoflat, that is, its curvature is identically null when elaborated via isomathematics and defined over isofields.

An elementary way of seeing the second features is to note that, under isotopies, we have the mutation of the Minkowskian coordinates while the corresponding unit is mutated by the inverse amount,

$$
\begin{gathered}
x_{k} \rightarrow \hat{x}_{k} \hat{I}_{k}=\frac{x_{k}}{n_{k}^{2}} \\
I_{k} \rightarrow \hat{I}_{k}=n_{k}^{2},
\end{gathered}
$$

thus preserving the original flatness.

In any case, isotopies must preserve the original axioms by central condition and technical realization. This means that, when properly treated, the isotopies of the Minkowski space must preserve the original flatness despite the dependence of the isometric on local coordinates.

\subsection{Lorentz-Santilli IsoSymmetry}

Thanks to the prior construction of the Lie-Santilli isotheory [20], the universal isosymmetry of all possible isoline elements (27) was constructed for the first time in only one page of Ref. [26]; it is today called the Lorentz-Santilli isosymmetry; it is characterized by the original symmetry plus the isotopic element (26); and can be written for isotransformations in the (3, 4)-plane (see Refs. [23] for the general case)

$$
\begin{aligned}
x^{\prime 3} & =\hat{\gamma}\left[x^{3}-\hat{\beta} \frac{n_{3}}{n_{4}} x^{4}\right], \\
x^{\prime 4} & =\hat{\gamma}\left[x^{4}-\hat{\beta} \frac{n_{4}}{n_{3}} x^{3}\right] .
\end{aligned}
$$

where

$$
\hat{\gamma}=\frac{1}{\sqrt{1-\hat{\beta}^{2}}}, \hat{\beta}=\frac{v / n_{3}}{c / n_{4}}
$$

As one can see, it is evident that the Lorentz-Santilli isosymmetry is locally isomorphic to the original symmetry by conception and realization. It is also evident that this local isomorphism is crucial for achieving compatibility of isogravitation with 20th century theories and for attempting a consistent grand unification of gravitation and electroweak interactions, as outlined below.

Following the original isotopies of the Lorentz symmetry 
$[26,27]$, systematic studies were done by the author on the isotopies of all most significant spacetime and internal symmetries. In fact, Ref. [29] was devoted to the isotopies $\hat{O}(3)$ of the rotational symmetry $O(3)$ to achieve the invariance of all topology preserving deformations of the sphere; Refs. [30,31] were devoted to the isotopies $\hat{S} U(2)$ of the $S U(2)$ spin symmetry; Ref. [32] presented for the first time the isotopies $\hat{P}(3.1)$ of the Poincaré symmetry $P(3.1)$ with the first proof of the universal invariance of all possible non-singular, Riemannian line elements; and Ref. [33] was devoted to the isotopies $P(3.1)$ of the spinorial covering of the Poincaré symmetry $P(3.1)$. Independent papers $[34,35]$ confirmed the universal character of the Lorentz-Santilli isosymmetry for the invariance of all infinitely possible symmetric line elements in $(3+1)$-dimensions.

\subsection{IsoGravitational IsoEquations}

Another important feature of isogravitation is that of preserving Einstein's field equations (2), although necessarily extended to forms (3)-(6) and reformulated on the Minkowski-Santilli isogeometry without curvature.

Along these lines, we have the isoequations for exterior gravitational problems

$$
\begin{aligned}
\hat{G}_{i j}= & \hat{R}_{i j}-\hat{\Xi}_{i j}(\hat{x}, \hat{v}) \hat{\times} \hat{R} / \hat{2}= \\
& =\hat{k} \widehat{\times} \hat{T}_{i j, e l m}(\hat{x}, \hat{v}),
\end{aligned}
$$

$$
\left(\widehat{\Xi}_{s t}^{\mu \nu} \widehat{\times} \hat{\Gamma}_{\mu} \widehat{\times} \hat{\partial}_{v}+\hat{\imath} \widehat{\times} \hat{m} \widehat{\times} \hat{c}\right) \hat{\times} \hat{\psi}=\left(\hat{\eta}_{s c h}^{\mu \nu} \hat{\gamma}_{\mu} \hat{\partial}_{v}+i m c\right) \hat{\psi}=0
$$

where $\Xi=\hat{\eta} \hat{I}, \hat{\Gamma}=\hat{\gamma} \hat{I}$ and the matrices $\hat{\gamma}$, known as the Dirac-Santilli IsoGamma matrices, are given by

$$
\begin{gathered}
\hat{\gamma}_{k}=\frac{1}{n_{k}}\left(\begin{array}{cc}
0 & \sigma_{k} \\
-\sigma_{k} & 0
\end{array}\right), \\
\hat{\gamma}_{4}=i \frac{1}{n_{4}}\left(\begin{array}{cc}
I_{2 \times 2} & 0 \\
0 & -I_{2 \times 2}
\end{array}\right),
\end{gathered}
$$

with anti-isocommutation rules [25]

$$
\left\{\hat{\gamma}_{\mu}, \hat{\gamma}_{v}\right\}=\hat{\gamma}_{\mu} T_{s t} \hat{\gamma}_{v}+\hat{\gamma}_{v} T_{s t} \hat{\gamma}_{\mu}=2 \hat{\eta}_{\mu \nu, s c h}
$$

As one can see, Eqs. (43) did indeed succeed in embedding gravitation in the Dirac equation, for which reason Santilli proposed the name of the Dirac-Schwartzchild IsoEquation [25]. It's expected physical relevance is evident, e.g., as the first description on scientific records of an electron within an intense gravitational field in the surface of the Sun or near the event horizon of a black hole.

In closing, we would like to honor the memory of Einstein, Podolsky and Rosen [36] for their view on the "lack of completeness of quantum mechanics" which was instrumental for the birth of hadronic mechanics and its applications. In fact operator isogravitation can be defined as an invariant non-unitary, axiom-preserving completion of relativistic quantum mechanics. under the condition

$$
m_{\text {grav }}=\int \widehat{T}_{00, e l m}(\hat{x}) \hat{\times} \hat{d} \hat{v}
$$

where one should note the dependence of the source on isocoordinates and isovelocities, as typical for electromagnetic source.

Consequently, the isometric is equally dependent on isocoordinates and isovelocities, $\Xi(\hat{x}, \hat{v})=\hat{\eta}(\hat{x}, \hat{v}) \hat{I}$, a property forbidden by the Riemannian geometry but readily permitted by the Minkowski-Santilli isogeometry due to the unrestricted functional dependence of the isometric.

We also have the broader isoequations for the interior isogravitational problem

$$
\begin{aligned}
& \hat{G}_{i j}=\hat{R}_{i j}-\hat{\Xi}_{i j}(\hat{t}, \hat{r}, \hat{v}, \hat{a}, \hat{\mu}, \hat{\tau}, \widehat{\omega}, \hat{\psi}, \ldots) \hat{\times} \hat{R} / \hat{2}= \\
& \hat{I}_{s c h}=\text { Diag. }\left[1,1,\left(1-\frac{r_{s c h}}{r}\right),\left(1-\frac{r_{s c h}}{r}\right)^{-1}\right] .
\end{aligned}
$$

or with the isogravitational characteristic quantities

$$
n_{r}^{2}=1-\frac{r_{s c h}}{r}, \quad n_{4}^{2}=\left(1-\frac{r_{s c h}}{r}\right)^{-1},
$$

where one should note the suggestive reformwhere one should note the suggestive reformulation of gravitational singularities in terms of the zeros of the space component of the isounit.

We now consider the isotopies of the Dirac equations introduced in Ref. [33], now called the Dirac-Santilli IsoEquations, and specialize then to the Schwartzchild metric

\subsection{Compatibility of IsoGravitation with 20th Century Theories}

The compatibility of isogravitation with 20th century sciences is direct and immediate. The compatibility of isogravitation with special relativity is immediately established by the fact that its universal isosymmetry is locally isomorphic to the conventional Poincaré symmetry. The compatibility of the physical laws of isogravitation with those of special relativity is then an immediate consequence.

The compatibility of isogravitation with the interior gravitational problem is established by the completely unrestricted functional dependence of the gravitational isometric. The compatibility of isogravitation with electromagnetism is established by the electromagnetic origin of the gravitational mass appearing in Eqs. (33).

The compatibility of isogravitation with quantum mechanics is inherent in the very notion of isotopies and it is used at the foundation of the very proposal of isogravitation [25]. The compatibility of isogravitation with grand unifications will be discussed in Section 7.

\subsection{IsoGravitational IsoAxioms}

The isotopies of the axioms of special relativity, today known as IsoAxioms, were initiated by Santilli in paper [26] of 1983; they received a first systematic formulation in 
monographs [37] of 1991; and they were finalized in monographs [23] of 1995 jointly with the discovery of the isodifferential calculus (see Ref. [41] for an independent review).

In works [23,26,37], the isoaxioms were specifically conceived and technically developed for quantitative treatments of relativistic interior dynamical problems, such as for the propagation of light within gaseous media (Figure 1), in which application they have received numerous experimental verifications (see, e.g., Refs. [11-15] and general review [41]).

The isoaxioms presented in Refs. [23,26,37] had no gravitational content. The application of the isoaxioms for a representation of gravity is presented for the first time in this paper under the proposed name of IsoGravitational IsoAxioms.

The presentation of this subsection is the most general possible for both the exterior and the interior gravitational problems characterized by a non-singular, symmetric isometric in $(3+1)$-dimension. This general formulation is merely achieved without any specification of the functional dependence of the isometric. In the verifications of the isogravitational isoaxioms of the next subsection, we will be forced to specify the isoaxioms to exterior or interior gravitational problems.

The first implication of the isotopies of special relativity is the abandonment of the speed of light in vacuum as the maximal causal speed in favor of a covering geometric notion. This is necessary for isogravitation because light is expected not to propagate within the hyperdense media inside planets or stars.

This occurrence is easily seen by specializing the isoline element (27) to the isolight isocone [23, 37]

$$
\left.\widehat{x}^{\hat{2}}=\frac{x_{k}^{2}}{n_{k}^{2}}-t^{2} \frac{c^{2}}{n_{4}^{2}}\right)=0,
$$

thus leading to the maximal Causal Speed $V_{\max }$ of IsoAxiom 5.1 below.

The remaining isoaxioms can be uniquely and unambiguously identified via a procedure parallel to the construction of the axioms of special relativity from the Poincaré symmetry [23,37].

The reader should be aware that isogravitation is generally inhomogeneous and anisotropic for both exterior and interior problems, as evidently intrinsic in the fact that the characteristic quantities $n_{k}$ of isoelement (27) generally have different values for different space directions.

These features are necessary for a more realistic representation of exterior and interior gravitational fields of planets such as Earth. Inhomogeneity and anisotropy are then easily represented thanks to the arbitrary functional dependence of the characteristic quantities of the Minkowski-Santilli isogeometry.

A consequence of the inhomogeneity and anisotropy of isogravitation is that the isoaxioms are presented for one given direction in space, hereon denoted with the sub-index $k$, since the change of space direction generally implies a change in the explicit value of the characteristic quantities.

ISOAXIOM 5.I: The maximal causal speed in a given space direction $k$ within an isogravitational field is given by

$$
V_{\max , k}=c \frac{n_{k}}{n_{4}}
$$

ISOAXIOM 5.II: The local isospeed of light within an isogravitational field is given by

$$
\hat{c}=\frac{c}{n_{4}}
$$

where $c$ is the speed of light in intergalactic spaces $\mathrm{w}$ where $c$ is the speed of light in intergalactic spaces without any gravitational field.

ISOAXIOM 5.III: The addition of isospeeds in the $k$-direction within an isogravitational field follows the isotopic law

$$
V_{t o t, k}=\frac{v_{1, k} / n_{k}+v_{2, k / n_{k}}}{1+\frac{v_{1, k} v_{2, k}}{c^{2}} \frac{n_{4}^{2}}{n_{k}^{2}}} .
$$

ISOAXIOM 5.IV: The isodilatation of isotime, the isocontraction of isolengths, theiso variation of mass with isospeed, and the mass-energy isoequivalence principle follow the isotopic laws

$$
\begin{gathered}
\Delta t^{\prime}=\widehat{\gamma_{k}} \Delta t, \\
\Delta \ell^{\prime}={\widehat{\gamma_{k}}}^{-1} \Delta \ell, \\
m^{\prime}=\widehat{\gamma_{k}} m, \\
E=m V_{\text {max }}^{2}=m c^{3} \frac{n_{k}^{2}}{n_{4}^{2}}
\end{gathered}
$$

where $\hat{\gamma}$ and $\hat{\beta}$ have values (32).

where $\hat{\gamma}$ and $\hat{\beta}$ have values (32).

ISOAXIOM 5.V: The frequency isoshift of light propagating within an isogravitational field in the $k$-direction follows the Doppler-Santilli isotopic law

$$
\omega_{e}=\omega_{o} \hat{\gamma}_{k}\left[1 \pm \frac{v / n_{k}}{c / n_{4}} \cos \alpha\right]
$$

where $\omega_{e}$ is the experimentally measured value, $\omega_{o}$ is the value at the origin, and we have ignored for simplicity the isotopies of trigonometry (see Refs. [23] for brevity).

A technical understanding of the isoaxioms requires a technical knowledge of isomathematics. In fact, the isoaxioms presented below are given by their projection from the Minkowski-Santilli isospace over an isofield with isounit (25) into the conventional Minkowski space over a conventional field with isounit 1 .

A main feature is that, when the isoaxioms are represented on isospace over isofields, they coincide with the conventional axioms of special relativity by conception and technical realization. In particular, the maximal causal speed $V_{\text {max }} \neq c$ solely occurs in the projection of the isoaxioms on Minkowski 
space because, at the isotopic level, the maximal causal speed is $c$ for all possible isogravitational problems.

\subsection{Verification of IsoGravitation for Exterior Problems without Source}

It It is important for the self-consistency of this paper to initiate the appraisal of isogravitation via its application to the exterior gravitational problem without source in order to verify that Einctein field equations (2) can indeed be consistently formulated on a Minkowski-Santilli isospace.

In fact, all consistent experimental verifications of general relativity also apply to isogravitation without source because, for its own conception and technical realizations, isotopic liftings preserve all original numerical values (for brevity, see ref. [23b] with particular reference to the proof that the maximal causal speed on Minkowski-Santilli isospaces on isofields is the conventional speed of light in vacuum c).

In particular, it is easy to see that Einstein's Equivalence Principle $[6,9]$ is maintained in its integrity for various independent reasons. First of all, the projection of isogravitation on the conventional Riemannian space over a conventional field coincides with Einstein gravitation with consequential trivial validity of Einstein';s Equivalence Principle. Additionally, the Equivalence Principle independently holds on the Minkowski-Santilli isospace over isofields by very conception of isotopies [23]. The verification of other serious experimental verifications of Einstein gravitation follows in the same way.

To verify the above general lines, let us assume the Schwartzchild metric (39) as a good approximation of the isometric for isoequations (33) for the case without source, and present the results for appraisal by interested readers.

Note that, under said assumption, we have the homogeneity and isotropy of the isogravitational field, thus eliminating the selected space direction $\$ \mathrm{k} \$$ of the general isoaxioms.

Note that, under said assumption, we have the homogeneity and isotropy of the isogravitational field, thus eliminating the selected space direction $k$ of the general isoaxioms.

Let us begin by recalling values (42) of the characteristic quantities for the Schwartzchild metric for which

$$
\begin{gathered}
\frac{\hat{v}}{\hat{c}}=\frac{v}{n_{r}} \frac{n_{4}}{c}= \\
=\frac{v}{1-r_{s c h} / r} \frac{1}{c\left(1-r_{s c h} / r\right)}= \\
=\frac{r / c}{\left(1-r_{s c h} / r\right)^{2}},
\end{gathered}
$$

and consequential expressions for the isogamma (32)

$$
\hat{\gamma}=\frac{1}{\sqrt{1-\hat{\beta}^{2}}}=\frac{1}{\sqrt{1-\frac{\hat{v}^{2}}{\hat{c}^{2}}}}=\frac{1}{\sqrt{1-\frac{v^{2}}{c^{2}} \frac{n_{4}^{2}}{n_{r}^{2}}}}=
$$

$$
\begin{gathered}
=\frac{1}{\sqrt{1-\left(\frac{v}{1-r_{s c h} / r}\right)^{2}\left(\frac{1}{c\left(1-r_{s c h} / r\right)}\right)^{2}}}= \\
=\frac{1}{\sqrt{1-\frac{v^{2} / c^{2}}{\left(1-r_{s c h} / r\right)^{4}}}} \approx \\
\approx \frac{1}{1-\frac{v / c}{\left(1-r_{s c h} / r\right)^{2}}}
\end{gathered}
$$

From the above values, we have the maximal causal speed in an isogravitational field

$$
c=c n 4=c(1-r s c h r)
$$

which evidently tends to zero at the event horizon.

We believe that this occurrence is a significant confirmation of isogravitation because it provided a most effective, quantitative representation of the impossibility of matter to escape from a black hole.

Similarly, we have the expression for the isospeed of light

$$
\hat{c}=\frac{c}{n_{4}}=c\left(1-\frac{r_{s c h}}{r}\right)
$$

which also tends to zero at the event horizon and expectedly thereafter.

We believe that this is another supporting feature of isogravitation because the speed of light decreases for about $100,000 \mathrm{~km} / \mathrm{sec}$ when propagating within water. It is then logical to assume that the speed of light is null when reaching the densest conceivable medium in the universe. The null value at the event horizon is also an effective way to represent the impossibility for light to escape from a black hole.

It should be noted that the conventional speed of light $c$ is । an invariant under the Lorentz-Santilli isosymmetry and related isogravitation because, e.g., the isosum of two light speeds $c$ does not reproduce $c$ as it is the case for special relativity.

However, isospeed (58) is indeed an isoinvariant because the isosum of two light isospeeds does indeed yield the light isospeed,

$$
V_{t o t, s}=\frac{c / n_{4}+c / n_{4}}{1+\frac{c^{2}}{c^{2}}}=\frac{c}{n_{4}}=c\left(1-\frac{r_{s c h}}{r}\right)
$$

The reader should be aware of the fact that isogravitation predicts that the speed of light $c$ in intergalactic spaces without any gravitational field is "bigger" then the speed of light $\hat{c}_{\text {earth }}$ measured on Earth, although for a very small amount,

$$
\hat{c}_{\text {earth }}=\frac{c}{n_{4}}=c\left(1-\frac{r_{s c h}}{r}\right)<c,
$$

By using isospeeds away from the observer, and values (42), we can write the first order approximation 


$$
\Delta t^{\prime}=\Delta t \hat{\gamma} \approx \frac{\Delta t}{1-\frac{v / c}{\left(1-r_{s c h} / r\right)^{2}}}
$$

which recovers the conventional time dilation of special relativity at a given distance $r$. However, the value of $\Delta t^{\prime}$ within a gravitational field ( $g r a v$ ) is predicted to be smaller than that for special relativity (sr),

$$
\Delta t_{\text {grav }}^{\prime}<\Delta t^{\prime}{ }_{s r}
$$

in such a way that time tends to zero at the event horizon, in full agreement with the behavior of the time component of the Schwartzchild metric (39),

$$
\operatorname{Lim}_{r \rightarrow s c h} \Delta t=0
$$

Similarly, we have the isolength isocontraction

$$
\Delta \ell^{\prime}=\frac{\Delta \ell}{\hat{\gamma}} \approx \Delta \ell 1-\frac{v / c}{\left(1-r_{s c h} / r\right)^{2}}
$$

which recovers the length contraction of special relativitywhich recovers the length contraction of special relativity for a given distance $r$. However, the value of $\Delta \ell^{\prime}$ in the presence of a gravitational field is predicted to be bigger than that of special relativity

$$
\Delta \ell_{\text {grav }}^{\prime}>\Delta \ell^{\prime}{ }_{s r}
$$

in such a manner that $\delta t^{\prime}$ tends to infinity at the event horizon

$$
\operatorname{Lim}_{r \rightarrow s c h} \Delta \ell=\infty
$$

also in full agreement with the space component of the Schwartzchild metric (39).

We also have from isoaxiom (52) the isovariation of mass in an isogravitational field

$$
m^{\prime}=\frac{m}{\sqrt{1-\frac{v^{2}}{c^{2}} \frac{n_{4}^{2}}{n_{r}^{2}}}} \approx \frac{m}{\sqrt{1-\frac{v^{2} / c^{2}}{\left(1-r_{s c h} / r\right)^{4}}}}
$$

illustrating the prediction based on the Schwartzchild metricthat the mass tends to zero at the event horizon.

Similarly, from the energy equivalence (53), we have in the vicinity of the event horizon

$$
\begin{aligned}
& E^{\prime}= m^{\prime} V_{\text {max }}^{2}=\frac{m}{\sqrt{1-\frac{v^{2} / c^{2}}{\left(1-r_{s c h} / r\right)^{4}}}} \mathrm{x} \\
& x c^{2}\left(1-\frac{r_{s c h}}{r}\right)^{4} \approx \\
& \approx m c^{2} \frac{\left(1-r_{s c h} / r\right)^{6}}{\left(1-r_{s c h} / r\right)^{2}-v / c}
\end{aligned}
$$

illustrating the prediction that the energy isoequivalence of a particle tends to zero at the event horizon much faster than that for the mass.
We believe that the above features are an important verification of the isoaxioms for various reasons. Firstly, the expectation that Newton's inertia and other laws are valid within a black hole is nowadays rejected by the vast majority of scientists. Secondly, any expectation that particles may experience inertia when constrained within the densest medium in the universe without any possibility of motion, is manifestly illogical. Thirdly, and perhaps most importantly, the limitation for the divergent increase of mass and energy within a black hole appears to be an important mechanism set by nature to prevent the achievement of infinite mass under which one single black hole would swallow the entire universe.

It should be stressed to prevent misrepresentations that the null limit of the mass at the event horizon is similar to the singularity of the Schwartzchild metric and solely occur for the case of field equations (2) without source. As indicated in the next subsection, the presence of a source of first order in magnitude, Eq. (4), appears to avoid both the null value of the mass and the singularity at 6 the event horizon by turning them into more realistic finite values.

For IsoAxiom 5.V, we have the Doppler-Santilli isoshift of the frequency of light within an exterior isogravitational field for the simple case of null aberration in the space $k$-direction

$$
\omega_{e} \approx \omega_{o}\left[1 \pm \frac{v}{c}\left(1-\frac{r_{s c h}}{r}\right)\right]
$$

clearly showing Santilli isoRedShift [11,37], namely a redshift of the entire spectrum of visible light without any relative motion between the light source and the origin of the gravitational field.

The energy lost by light for the isoredshift when traversing a gravitational field is expected to be one of the continuous sources of energy needed for the Cosmic Background Radiation to exist in view of its weakness, in addition to the energy originating from the de-excitation of hydrogen atoms in intergalactic spaces when hit by light [11-15] which appears to be an additional source of the energy needed to maintain in time the Cosmic Backgrtound Radiation [11-15].

Note also that all frequencies of visible light become identically null at the event horizon. This feature is necessary for compatibility with the null value of the speed of light at the event horizon, thus confirming the plausible expectation that the conventional notion of electromagnetic waves becomes meaningless within the densest media existing in the universe. Needless to say, the energy lost by light to the event horizon is absorbed by the black hole.

In conclusion, to our best understanding at this writing, the predictions of isogravitational isoaxioms for matter appear to be supported by the behavior of the isotopic reformulation of the Schwartzchild metric, although more studies are evidently needed to achieve any conclusion due to the complexity of the problem and our rather limited final knowledge of black holes.

\subsection{Verification of IsoGravitation for Exterior Problems with Source}

As indicated earlier, the Schwartzchild metric (39) has a just place in the history of gravitation because it achieved for 
the first time a geometric understanding of gravitational singularities, besides other advances.

However, the Schwartzchild metric remains a first approximation of a rather complex physical reality because local infinities cannot exist in the universe as a condition for its continued existence.

Following decades of studies on the covering of the Schwartzchild metric suitable to avoid local infinities, the author has found no other consistent approach than that allowed by a first-order electromagnetic source in the r.h.s. of the field equations according to Eqs. (33).

This raises the question as to whether Einstein's Equivalence Principle also holds for exterior isogravitation with a source. Einstein supporters quickly voice their opinion that this is not the case for the intent of invalidating isogravitation. However, serious science is far from these unsubstantiated personal opinions because the problem is rather complex indeed and, to avoid a prohibitive length, it will be studied by the author in a subsequent paper.

At this moment, we limit ourselves to the indication that, apparently, the introduction of a source in the gravitational field equations implies numerical contributions in the verification of the Equivalence Principle well within experimental errors. Consequently, the introduction of a source does not invalidate the Equivalence Principle on serious scientific grounds until proved so with detailed calculations published in serious refereed journals.

The needed solution is scheduled for detailed studies in a subsequent paper. For the completeness of this paper, we limit ourselves to indicate that an approximate solutions of Eqs. (33) can be written

$$
\begin{gathered}
d s^{2}=r^{2}\left(d \theta^{2}+\sin ^{2} d \theta^{2}+d \phi^{2}\right)+ \\
+\left(1-\frac{r_{s c h}+S(r, v)}{r}\right)^{-1} d r^{2}- \\
-\left(1-\frac{r_{s c h}+S(r, v)}{r}\right) d t^{2} \equiv \\
\equiv \widehat{T}_{s c h} \times \eta \equiv \hat{\eta}_{s c h},
\end{gathered}
$$

with characteristic quantities

$$
\begin{gathered}
=1-\frac{r_{s c h}+S(r, v)}{r}, n_{4}^{2}= \\
=\left(1-\frac{r_{s c h}+S(r, v)}{r}\right)^{-1},
\end{gathered}
$$

whose limit for $r \rightarrow 0$ (rather than for $r \rightarrow s c h$ ) is such to avoid local singularities, e.g., of the type

$$
\begin{gathered}
\operatorname{Lim}_{r \rightarrow 0}\left(1-\frac{\hat{v}}{\hat{c}}\right)= \\
=\operatorname{Lim}_{r \rightarrow 0}\left[1-\frac{r / c}{1-\frac{r_{s c h}+S(r, v)}{r}}\right]=
\end{gathered}
$$

$$
=N \neq 0, \quad N<\infty
$$

and the numerical value of $N$ evidently requires the consideration of a specific black hole.

It then follows that isogravitational isoequations (33) with a first-order electromagnetic source recover all main historical results achieved by the Schwartzchild's metric, with the elimination of singularities that are not expected to exist in nature.

As a first illustration, the expected behavior of the isotime isodilation (61) acquires the form

$$
\begin{aligned}
\operatorname{Lim}_{r \rightarrow 0} \Delta t^{\prime} & \approx \operatorname{Lim}_{r \rightarrow 0} \frac{\Delta t}{\left(1-\frac{\hat{v}}{\hat{c}}\right)^{2}}= \\
& =\frac{\Delta t}{N^{2}}>0,
\end{aligned}
$$

thus eliminating the singularity in time of the Schwartzchild metric (39)

Similarly, for the isolength isocontraction we have

$$
\begin{aligned}
\operatorname{Lim}_{r \rightarrow 0} \Delta \ell & \approx \operatorname{Lim}_{r \rightarrow 0} \Delta \ell\left(1-\frac{\hat{v}}{\hat{c}}\right)^{2}= \\
& =N^{2} \Delta \ell<\infty,
\end{aligned}
$$

thus eliminating jointly the local singularity of the Schwartzchild metric for the space component.

Similar corrections occur for the remaining physical quantities studied in the preceding subsection, as the reader can verify.

Note the truly crucial role of the first-order nature of the electromagnetic source, that is, such to represent the entire gravitational mass, Eq. (34). In fact, the standard consideration of the total electromagnetic characteristics of a body leaves Schwartzchild's singularities completely unaffected since their contribution to the gravitational field is of the order of $10^{-30}$ or less.

In conclusion, we can state that the inclusion in the r.h.s. of the field equation of a first order source of electromagnetic character, essentially along Einstein's own intuition, besides achieving compatibility of gravitation with electrodynamics, does indeed offer realistic possibilities of avoiding local infinities in the universe, with ensuing significant advances in various gravitational problems.

\subsection{Verification of IsoGravitation for the Interior Problem}

Contrary to isogravitation, Einstein gravitation cannot even formulate interior gravitational problems in any realistic way, e.g., due to the inability to represent a locally varying speed of light. In this case there is the loss of credibility for Einstein supporters who even mention experimental verifications of Einstein gravitation, for the evident reason that we have no direct experimental tests in the interior of the Sun or planet.

The interior character of the Doppler-Santilli isolaw has been extensively studied in Refs. [11-15]. We hereby limit ourselves to consider the interior gravitational case of light passing through the Sun chromosphere.

In this case, the characteristic $n$-quantities have a functional dependence on the speed $v$, the distance $d$ 
covered within the physical medium, etc. thus admitting the expansion in the travwersed distance $d$ by light within the medium

$$
\begin{array}{r}
n_{4} n_{k} \approx 1 \pm \\
\frac{n_{4}}{n_{k}} \approx 1 \pm \frac{c}{v} H d,
\end{array}
$$

where $H$ is the Hubble constant with resulting Doppler-Santilli isoshift law [11]

$$
\omega_{e} \approx \omega_{0}[1 \pm v c(1 \pm H d)]
$$

Measurements [11-15] have established that, for a sufficiently dense chromosphere (or for a sufficiently long travel $\mathrm{d}$ in a thin atmosphere), the conventional Doppler term is ignorable, e.g., for the case of earth's rotation, and the Hubble term becomes dominant, resulting in the Hubble law for the cosmological shift

$$
z= \pm H d
$$

which is uniquely and unambiguously characterized by the Poincaré-Santilli isosymmetry.

We hereby add, apparently for the first time, the extended version of the Doppler-Santilli isolaw within a transparent physical medium with the inclusion of a strong isogravitational, field from isoaxiom (64)

$$
\omega_{e} \approx \omega_{0}\left[1 \pm \frac{v}{c}\left(1-\frac{r_{s c h}}{r}\right) \pm H d\right) .
$$

As one can see, the isoredshift caused by isogravitation in the vicinity of the event horizon is dominant over all others, as expected.

We believe that the above features provide a significant additional verification of isogravitation at large, and of its isoaxioms in particular.

In conclusion, we can safely state that isogravitation does indeed allow meaningful models of interior gravitational problems that are notoriously impossible for Einstein gravitation.

It should be noted that these interior gravitational models are reached via the same axioms of exterior problems under the uncompromisable condition that the metric has an unrestricted functional dependence on all possible interior local variable, which dependence is solely admitted at this writing by isogeometries.

\section{Rudiments of IsoDual IsoGravitation for AntiMatter}

Despite all the above advances, attempts at an axiomatically consistent grand unification of electroweak and gravitational interaction continued to be inconsistent and not worth their presentation in a scientific paper, because Einstein gravitation, as well as isogravitation, solely apply for matter-bodies, thus preventing any consistent unification with electroweak theories that are bona-fide theories of particles and antiparticles.

A solution of the latter problem required the construction of yet another new mathematics, specifically conceived for the classical representation of neutral (or charged) antimatterbodies.

The transition from matter to antimatter required the new mathematics to be anti-isomorphic in general and anti-Hermitean in particular, to isomathematics, as a condition to be consistent with charge conjugation and experimental data, including matter-antimatter annihilation.

Following numerous failed attempts, when being at he Department of Mathematics of Harvard University in the early 1980s, the author finally succeeded in identifying the needed mathematics, called isodual mathematics and denoted with the upper symbol $d$.

In view of the above aspects, Santilli constructed the isodual image of 20th century mathematics and quantum mechanics under the condition of admitting the isodual unit

$$
I^{d}=-1
$$

at all its mathematical and physical levels [40].

The above studies remained grossly insufficient to initiate studies on possible grand unifications due to the need of the anti-isomorphic image of isomathematics for antimatter whose need emerges even stronger from the model of isogravitation presented in this paper.

The latter mathematics was built via the systematic application of the following isodual map

$$
\begin{gathered}
\hat{I}(t, r, p, \mu, \tau, v, \psi, \ldots) \rightarrow \hat{I}^{d}= \\
-\hat{I}^{\dagger}\left(-t^{\dagger},-r^{\dagger},-v^{\dagger},-a^{\dagger},-\mu^{\dagger},-\tau^{\dagger},-v^{\dagger},-\psi^{\dagger}, \ldots\right)
\end{gathered}
$$

to the totality of quantities and the totality of their operations used for matter.

The resulting new mathematics is today known as Santilli isodual isomathematics and includes isodual isonumbers, isodual isofunctions, isodual isodifferential calculus, isodual isoalgebras, isodual isogeometries, etc. (see monograph [40] for a comprehensive study and Ref. [41] for an independent general review).

Following the construction of the isodual isomathematics it was necessary to construct the isodual image of classical and operator theories, with particular reference to the isodual Lorentz-Santilli isosymmetry and the axiomatically consistent classical representation of the gravitational field of neutral (or charged) antimatter-bodies. The compatibility of the emerging isodual theory of antimatter with experiental data was assured by the equivalence of the isodual map with charge conjugation (for brevity, one may inspect monograph [23]).

\section{Rudiments of IsoGrandUnification}

In our view, a most important implication of the search for axiomatically consistent grand unifications is the shift from the description of gravitation to a study of its origin. In fact, Ref. [16] is crucially dependent on the abandonment of the standard "unification" of gravitation and electromagnetic interactions in favor of their "identification" under appropriate 
field equations.

Ref. [16] also submitted experiments for the possible laboratory creation of a measurable gravitational field that appears feasible nowadays thanks to the availability of highly sensitive detectors, such as those based on neutron interferometry.

Only following the above scientific journey the author was finally in a position to present at the 1997 Marcel Grossmann Meeting in Gravitatio, a grand unification of electroweak and gravitational interactions with the inclusion of matter and antimatter at all classical and operator levels [38] (see also Ref. [39]).

The emerging grand unification essentially consistent in the embedding of gravitation in the gravitational isounit of electrostatic interactions under the universal isospinorial covering $P(3.1)$ of the Poincaré-Santilli isosymmetry $\hat{P}(3.1)$ the selected isotopic image of the selected gauge symmetry $g$ for matter and their isodual for antimatter

$$
\hat{S}=\{\hat{P}(3.1) \times \hat{G}\} \times\left\{\hat{P}^{d}(3.1) \times \hat{G}^{d}\right\}
$$

which is the isosymmetry of the Dirac-Santilli isoequations (43) [33] and which, rather intriguingly, emerges as the isosymmetry of the universe at the limit of equal amounts of matter and antimatter (see monograph [40] for brevity).

Of course, we do not know whether the abovegrand unification is verified in nature, but we believe that the studies reported in this paper have provided at least much needed new vistas in gravitation [41] for further advances by interested colleagues.

To follow Albert Einstein teaching for powerful self-criticism, we note that the dynamics of test masses in a gravitational field is fully reversible in time. By contrast, the dynamics of a black holes is strictly irreversible over, since we are dealing with a one way absorption of matter and light.

By remembering that isomathematics and related isomechanics are reversible over time, \, a more accurate description of black holes may require a covering of isogravitation constructed via genomathematics with a Lie-admissible (rather than a Lie-isotopic) structure and related genogeometries with non-symmetric genometrics as a condition to embed irreversibility in the ultimate mathematical and physical structures [19,23].

All in all, the studies presented in this paper confirm that physics is a discipline that will never admit final theories.

\section{Acknowledgments}

The author would like to thank the organizers of the Eight Marcel Grossmann Meeting in Gravitation, held at the Hebrew University, Jerusalem, June 22 to 27, 1997, for having my talk presented by the session chairman and publishing my paper [34] in the proceedings despite my being incapacitated to attend.

\section{References}

[1] H.A.Lorentz, Amst. Proc. Vol. 6, 809 (1904).
[2] H.Poincare', Compte Rendues, Paris Vol.140, 1504 (1905).

[3] A.Einstein, Ann. Phys. Vol. 17, 891 (1905).

[4] H.Minkowski, Nachr. Ges. Wiss. Gottingen Vol. 43, (1908).

[5] R M. Santilli, 2014 Keynote Lectures "IsoLinear, IsoInvariant IsoRelativity"

http://www.world-lecture-series.org/isorelativity-2014-i http://www.world-lecture-series.org/isorelativity-2014-ii http://www.world-lecture- series.org/isorelativity-2014-iii

[6] C. W. Misner, K. S. Thorne and J. A. Wheeler, Gravitation, W. H. Freeman and Company (1973)

[7] R. M. Santilli, "Historical Insufficiencies of General Relativity and their possible resolution via Isogravitation, Galilean Electrodynamics, Vol 17 No. 3, page 43(2006) www.santillifoundation.org/docs/Incons.GravFinalGED-I.pdf

[8] J. V. Kadeisvili, "Obscurantism on Ein- stein's gravitation," http://www.santilligravitation.php

[9] C. M. Will, "The Confrontation between General Relativity and Experiment," Liv- ing Rev. Relativity, Vol. 9, p. 3 (2006).

[10] S. Beeson, Steven and J. W. Mayer, Pat- terns of Light Chasing the Spectrum from Aristotle to LEDs, Springer (2008)

[11] R. M. Santilli, "Experimental Verifications of IsoRedShift with Possible Absence of Universe Expansion, Big Bang, Dark Matter, and Dark Energy," The Open Astronomy Journal 3, 124 (2010), http://www.santilli- foundation.org/docs/Santilliisoredshift.pdf

[12] R. M. Santilli, "Experimental Verification of IsoRedShift and its Cosmological Implications," AIP Proceedings Vol. 1281, pp. 882-885 (2010)http://www.santillifoundation.org/docs/Isoredshift-Letter.pdf

[13] G. West and G. Amato, "Experimental Confirmation of Santilli's IsoRedShift and IsoBlueShift," Journal of Computational Methods in Sciences and Engineering, 12, 169 (2012),http://www.santillifoundation.org/docs/Confirmation-IRS-IBS.pdf

[14] R. M. Santilli, G. West and G. Amato. "Experimental Confirmation of the IsoRedShift at Sun at Sunset and Sunrise with Consequential Absence of Universe Expansion and Related Conjectures, " Journal of Computational Methods in Sciences and Engineering, 12, 165 (2012) http://www.santillifoundation.org/docs/Confirmation-sun-IRS.pdf

[15] H. Ahmar, G. Amato, J. V. Kadeisvili, J. Manuel, G. West, and O. Zogorodnia, "Additional experimental confirmations of Santilli's IsoRedShift and the conse- quential expected absence of universe expansion," Journal of Computational Methods in Sciences and Engineering, 13, 321 (2013),http://www.santillifoundation.org/docs/IRS-confirmations- 212.pdf

[16] R. M. Santilli, "Partons and Gravitation: some Puzzling Questions," (MIT) Annals of Physics, Vol. 83, 108-157 (1974), http://www.santilli- foundation.org/docs/Santilli-14.pdf.

[17] P. Freud, Ann. Math. 40 (2), 417 (1939)

[18] H. Rund, Algebras, Groups and Geome- tries Vol. 18, 267 (1991). 
[19] R. M. Santilli, "Lie-admissible invari- ant representation of irreversibility for matter and antimatter at the classical and operator levels," Nuovo Cimento B bf 121, 443 (2006), http://www.santilli-

foundation.org/docs/Lie-admiss-NCB-I.pdf

[20] M. Santilli, Foundations of Theoretical Mechanics, Volume I (1978) [20a], and Volume II (1982) [20b], Springer-Verlag, http://www.santilli- foundation.org/docs/Santilli-209.pdf http://www.santilli- foundation.org/docs/santilli-69.pdf

[21] R. M. Santilli, 'Isonumbers and Genon- umbers of Dimensions $1,2,4,8$, their Isoduals and Pseudoduals, and "Hidden Numbers" of Dimension 3, 5, 6, 7," Algebras, Groups and Geometries Vol. 10, 273 (1993), http://www.santillifoundation.org/docs/Santilli-34.pdf

[22] R. M. Santilli, "Nonlocal-Integral Iso- topies of Differential Calculus, Mechanics and Geometries," in Isotopies of Contemporary Mathematical Structures, P. Vetro Editor, Rendiconti Circolo Matematico Palermo, Suppl. Vol. 42, 7-82 (1996), $\mathrm{http}: / / w w w . s a n t i l l i-$ foundation.org/docs/Santilli-37.pdf

[23] R. M. Santilli, Elements of Hadronic Mechanics, Vol. I (1995) [23a], Vol. II 91995) [23b], Academy of Sciences, Kiev, available in free pdf downloads from, http://www.santillifoundation.org/docs/Santilli-300.pdf foundation.org/docs/Santilli-301.pdf

[24] R. M. Falcon Ganfornina and J. Nunez Valdes, Founsations of Santilli Isotopies, original verison in Spanish, International Academic Press (2001), english translations, Algebras, Groups and Geometries Vol. 32, pages 135-308 (2015),http://www.i-b-r.org/docs/Aversa-translation.pdf

[25] S. Georgiev, Foundations of the IsoDiffer- ential Calcuilus, Volumes I, II, III, Nova Scientific Publishers (2014 and 2015).

[26] R. M. Santilli, "Isotopic quantization of gravity and its universal isopoincare' symmetry" in the Proceedings of "The Seventh Marcel Grossmann Meeting in Gravitation, SLAC 1992, R. T. Jantzen, G. M. Keiser and R. Ruffini, Editors, World Scientific Publishers pages 500-505(1994), http://www.santilli- foundation.org/docs/Santilli-120.pdf

[27] R. M. Santilli, "Lie-isotopic Lifting of Spe- cial Relativity for Extended Deformable Particles," Lettere Nuovo Cimento Vol. 37, $545 \quad$ (1983), http://www.santillifoundation.org/docs/Santilli-50.pdf

[28] R. M. Santilli, "Lie-isotopic Lifting of Unitary Symmetries and of Wigner's Theorem for Extended and Deformable Particles," Lettere Nuovo Cimento Vol. 38, 509 (1983), http://www.santilli- foundation.org/docs/Santilli-51.pdf

[29] R. M. Santilli, "Isominkowskian Geom- etry for the Gravitational Treatment of Matter and its Isodual for Antimatter," Intern. J. Modern Phys. D Vol. 7, 351 (1998), http://www.santilli- foundation.org/docs/Santilli-35.pdf

[30] R. M. Santilli, "Isotopies of Lie symme- tries, I and II" Hadronic J. 8, $36 \quad$ (1985), http://www.santillifoundation.org/docs/santilli-65.pdf
[31] R. M. Santilli, "Isotopic Lifting of the SU(2) Symmetry with Applications to Nuclear Physics," JINR rapid Comm. Vol. 6. 24-38 (1993), http://www.santillifoundation.org/docs/Santilli-19.pdf

[32] R. M. Santilli, "Isorepresentation of the Lie-isotopic SU(2) Algebra with Ap- plication to Nuclear Physics and Local Realism," Acta Applicandae Mathemati- cae Vol. 50, 177 (1998), http://www.santillifoundation.org/docs/Santilli-27.pdf, see also R. M. Santilli, "Nonlinear, Nonlocal and Noncanonical Isotopirs of the Poincare' Symmetry," Moscow Phys. Soc. Vol. 3, 255 (1993),

foundation.org/docs/Santilli-40.pdf http://www.santilli-

[33] R. M. Santilli, "Recent theoretical and experimental evidence on the synthesis of the neutron," Communication of the JINR, Dubna, Russia, No. E4-93-252 (1993), published in the Chinese J. System Eng. and Electr. Vol. 6, 177 (1995), http://www.santilli- foundation.org/docs/Santilli-18.pdf

[34] J. V. Kadeisvili, "Direct universality of the Lorentz-Poincare'-Santilli isosymme- try for extended-deformable particles, arbitrary speeds of light and all possible spacetimes" in Photons: Old problems in Light of New Ideas, V. V. Dvoeglazov Editor Nova Science (2000, http://www.santilli- foundation.org/docs/Santilli-25.pdf

[35] A. K. Aringazin and K. M. Aringazin, "Universality of Santilli's iso-Minkowskian geometry" in Frontiers of Fundamental Physics, M. Barone and F. Selleri, Editors Plenum 91995),

foundation.org/docs/Santilli-29.pdf http://www.santilli-

[36] J. S. Bell, Speakable and Unspeakable in Quantum Mechanics, Cambridge Univer- sity Press (1987).

[37] R. M. Santilli, Isotopic Generalizations of Galilei and Einstein Relativities, Vols. I [37a] and II [37b] (1991), International Academic Press http://www.santillifoundation.org/docs/Santilli-01.pdf http://www.santillifoundation.org/docs/Santilli-61.pdf

[38] R. M. Santilli, "Unification of gravitation and electroweak interactions" in the proceedings of the Eight Marcel Grossmann Meeting in Gravitation, Israel 1997, T. Piran and R. Ruffini, Editors, World Scientific, pages 473-475 (1999), http://www.santilli- foundation.org/docs/Santilli-137.pdf

[39] R. M. Santilli, "Isotopic grand unification with the inclusion of gravity," Found. Phys. Letters 10, 307 (1997), http://www.santilli- foundation.org/docs/Santilli-04.pdf

[40] R. M. Santilli, Isodual Theory of Antimat- ter with Application to Antigravity, Grand Unification and the Spacetime Machine, Springer 2001 , http://www.santilli-foundation.org/docs/santilli-79.pdf

[41] I. Gandzha and J. Kadeisvili, New Sciences for a New Era: Mathemati- cal, Physical and Chemical Discoveries of Ruggero Maria Santilli, Sankata Printing Press, Nepal (2011), http://www.santilli- foundation.org/docs/RMS.pdf. 